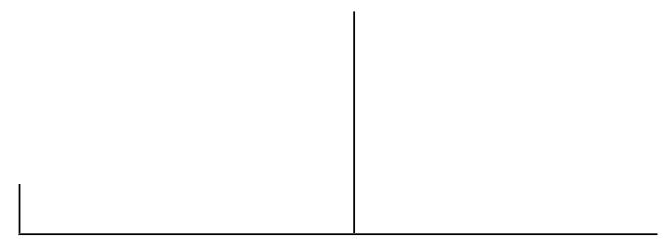

Rev. Latinoam. Psicopat. Fund., São Paulo, 16(1),42-55, mar. 2013

\title{
Resiliência e psicanálise: aspectos teóricos e possibilidades de investigação
}

\author{
Stela Araúio Cabral \\ Daniela Centenaro Levandowski
}

A resiliência é um conceito do campo da saúde que analisa os recursos saudáveis do indivíduo frente a situações adversas. Discutir suas possibilidades em diferentes contextos teóricos e clínicos constitui um debate construtivo e necessário. O presente trabalho apresenta os pressupostos deste conceito e discute as possibilidades de análise da resiliência segundo a perspectiva psicanalítica. Essa perspectiva investiga a resiliência através da análise dos aspectos intrapsíquicos do indivíduo, possibilitando uma nova interpretação do conceito.

Palavras-chave: Resiliência, psicanálise, aspectos intrapsíquicos, saúde mental 


\section{Conceituando resiliência}

O conceito resiliência integra o campo da saúde mental e constitui uma preocupação central da clínica contemporânea (Anault, 2006), pautada na saúde e nos recursos do indivíduo. Entretanto, a vulnerabilidade ainda se apresenta como modelo dominante de investigação nessa área. Esse modelo está vinculado à patologia, aos problemas mentais que podem causar danos ao funcionamento do indivíduo (Anault, 2006; Bertrand, 2006; Laranjeira, 2007).

$\mathrm{O}$ termo resiliência é originário da Física e designa a capacidade elástica que certos corpos têm de voltarem ao estado anterior após sofrerem uma deformação (Cyrulnik, 2004). Como construto psicológico, pode ser definido como a adaptação pessoal bem-sucedida a situações adversas. Desta fazem parte tanto os aspectos intrapsíquicos, ou seja, os recursos internos do indivíduo, como os aspectos externos, por exemplo, o meio social e afetivo (Laranjeira, 2007). A resiliência corresponde a uma resposta complementar à vulnerabilidade, que leva à flexibilidade e à adaptação, contrapondo-se à ideia de invulnerabilidade e invencibilidade referida em estudos iniciais sobre o tema (Golse, 2006; Laranjeira, 2007, Lemay, 2001).

Os estudos, em geral, investigam os riscos e os comportamentos de risco em situações consideradas adversas, não priorizando a investigação da resiliência em si (Laranjeira, 2007; Lindström, 2001). Os fatores de risco ou adversidades constituem aspectos que podem contribuir para a aquisição de psicopatologias, causando prejuízos ao desenvolvimento do indivíduo (Sapienza \& Pedremônico, 2005). Por outro lado, é a partir da "resistência" do indivíduo a esses fatores que se poderia avaliar a resiliência. 
A investigação da resiliência está relacionada à análise de vários aspectos individuais, como aqueles associados à genética, idade, fase do desenvolvimento, sexo, experiência e histórico de vida. Também são considerados os fatores contextuais, como o suporte social, o nível sociocultural e o ambiente. Ressalta-se que tal investigação prioriza a identificação de fatores de proteção, que podem modificar ou alterar a resposta do indivíduo frente a uma situação adversa (Lindström, 2001; Souza \& Cerveny, 2006).

Diante do exposto, pensa-se que discutir as diferentes contribuições teóricas e clínicas e os limites do modelo de resiliência no campo da saúde constitui um debate contemporâneo construtivo (Anault, 2006). Sendo assim, o presente estudo objetiva tecer algumas considerações sobre o conceito resiliência, apresentando os principais contextos teóricos de sua abordagem, bem como sua possível conceituação e investigação segundo a perspectiva psicanalítica.

\section{A resiliência em diferentes contextos teóricos}

A investigação da resiliência pela psicologia encontrou respaldo inicial na psicologia cognitivo-comportamental e na psicologia social e ecossistêmica (Bertrand, 2006), destacando-se as abordagens da psicologia positiva (Seligman \& Csikszentmihalyi, 2000) e do modelo bioecológico de desenvolvimento humano (Bronfenbrenner \& Morris, 1998).

Os estudos da psicologia positiva (Seligman \& Csikszentmihalyi, 2000) encontram-se inicialmente pautados na psicologia humanista, focados no desenvolvimento saudável e positivo dos aspectos psicológicos, biológicos e sociais do indivíduo. A psicologia positiva investiga a resiliência através dos aspectos positivos do indivíduo diante de situações adversas. A terapia positiva, por sua vez, tem como objetivo fortalecer os traços positivos e os comportamentos adaptativos frente a tais situações (Paludo \& Koller, 2007).

Esse conceito também é utilizado na psicologia cognitivo-comportamental, pela análise dos processos mentais conscientes presentes nos processos de enfrentamento (coping) de situações adversas (Bertrand, 2006). Para tanto, utiliza-se de instrumentos como escalas e questionários para classificar as atitudes e reações frente à adversidade, especialmente as estratégias de enfrentamento utilizadas, e assim reconhecer os aspectos positivos do indivíduo. A utilização de tais instrumentos objetiva a avaliação e a classificação das virtudes e forças pessoais (Paludo \& Koller, 2007).

Já o modelo bioecológico de desenvolvimento humano (Bronfenbrenner \& Morris, 1998) é pautado na psicologia social e ecossistêmica e investiga a 
resiliência através das interações do indivíduo com o meio no qual ele está inserido, a partir da análise de quatro núcleos: o processo, a pessoa, o contexto e o tempo. O processo corresponde à interação do ser humano com as pessoas, objetos e símbolos que fazem parte do seu ambiente próximo. O segundo núcleo, a pessoa, é investigado por meio das caraterísticas biopsicológicas e de suas interações com o ambiente. $O$ contexto constitui o terceiro núcleo, examinado pela interação entre o indivíduo e seu ambiente. Por fim, o tempo analisa o efeito de mudanças e de processos contínuos que ocorrem no desenvolvimento humano ao longo do ciclo vital (Paludo \& Koller, 2007).

Ainda cabe referir os estudos de resiliência na teoria sistêmica, divulgados por Delage (2002) e Walsh (1996). A resiliência familiar se apresenta como a capacidade coletiva de construir, organizar e/ou reorganizar-se diante de circunstâncias difíceis. A família surge como meio de sustentação quando um ou mais de seus membros vivenciam situações adversas. Essa abordagem busca analisar, através do trabalho terapêutico, as capacidades resilientes presentes na inter-relação da pessoa atingida com o grupo familiar, por meio do apoio do sistema familiar (Delage, 2002). Também fazem parte os processos de comunicação, as crenças e a organização familiar na superação de desafios, auxiliando no crescimento e na transformação pessoal do indivíduo (Walsh, 1996; Yunes, 2003). Os estudos de resiliência familiar são recentes e inserem este construto no âmbito grupal. Assim, apresentam uma proposta que extrapola a investigação individual, ainda a forma mais utilizada de investigação deste conceito (Yunes, 2003; Yunes \& Szymanski, 2006).

Diante do mencionado, percebe-se que a resiliência apresenta-se como um conceito contemporâneo de saúde ainda em construção. Sua investigação pode variar de acordo com a proposta de estudo e do referencial teórico utilizado. Considera-se relevantes todas as contribuições na descoberta das especificidades e aplicações deste conceito, que se insere em um novo paradigma de saúde, que privilegia os recursos saudáveis do indivíduo.

Outro ponto a destacar são as especificidades das abordagens em sua investigação. Estas abrangem desde os aspectos individuais, como o fortalecimento dos traços positivos do indivíduo, até a sua relação com o seu meio social, comunitário e familiar, caracterizando a resiliência como um processo. Da mesma forma, percebe-se que todas as abordagens investigam a resiliência considerando aspectos conscientes do indivíduo frente a situações adversas, especialmente suas percepções. Portanto, evidencia-se que sua investigação em uma abordagem psicodinâmica ainda é pouco explorada, apesar de se mostrar bastante adequada para o trabalho clínico nessa perspectiva. Nesse sentido, discute-se, a seguir, algumas contribuições para a conceituação e a investigação da resiliência na abordagem psicanalítica. 


\section{Resiliência e psicanálise: um diálogo possível?}

Embora sendo um conceito da psicologia contemporânea, falar em resiliência na psicanálise gera controvérsias e dúvidas no meio psicanalítico e fora dele, uma vez que, conforme comentado anteriormente, este conceito está associado a outros contextos teóricos e clínicos. Portanto, é comum observar reticência acerca do uso do termo no campo psicanalítico (Bertrand, 2006).

De fato, a psicanálise ainda está se apropriando do que denomina noção de resiliência, buscando defini-la de acordo com os seus pressupostos teóricos próprios. Este conceito não é utilizado massivamente nessa perspectiva (Bertrand, 2006). Pesam sobre isso, preferencialmente, questões epistemológicas, pois resiliência não é um conceito do corpo teórico da psicanálise. No entanto, dialogar com conceitos de outros campos do conhecimento, sem renunciar aos princípios da teoria, constitui um desafio enriquecedor para essa disciplina (Golse, 2003).

Além disso, a contemporaneidade leva à produção de novos conhecimentos e a psicanálise, acompanhando esse movimento, mantém a curiosidade e o interesse em conhecer novas formas de entendimento e investigação do psiquismo. Assim, também se apresenta sensível à contribuição de novos conceitos, que buscam reorganizar esse campo de saber, com o objetivo de melhor compreender o cuidado ao ser humano. Nesse sentido, situa-se a noção de resiliência (Bourguignon, 2006).

A evidência de tal debate se fez presente no evento internacional Résilience: Réparation, Élaboration ou Création, realizado no ano de 2006, em Toulouse, França. O evento teve por objetivo refletir acerca do entendimento psicanalítico desse conceito. Dessa forma, pode-se referir que essa proposta mantém o espírito criativo e aberto desta perspectiva teórica, que foi tão bem ilustrado por seu fundador, Sigmund Freud.

Interessante notar que a noção de resiliência está implicitamente presente na psicanálise a partir de algumas referências de Freud, obviamente sob outra denominação. Em seus estudos (Freud, 1920), ele mencionou a realidade psíquica e sua relação com a realidade exterior, supondo a existência de aparelhos de proteção na psique, que funcionariam como filtros contra as excitações exteriores excessivas. Esta ideia já estava presente em seu "Projeto para uma psicologia científica" (1895) e, de certa forma, aponta que a resiliência não se apresenta como uma noção totalmente estranha à psicanálise (Tychey \& Lighezzolo, 2006).

Desse modo, o referencial psicanalítico investiga o processo resiliente através da análise do funcionamento intrapsíquico do indivíduo. O processo resiliente é percebido após o trauma (situação adversa), por meio do efeito psíquico gerado pelo mesmo, chamado traumatismo. A psicanálise investiga, então, o traumatis- 


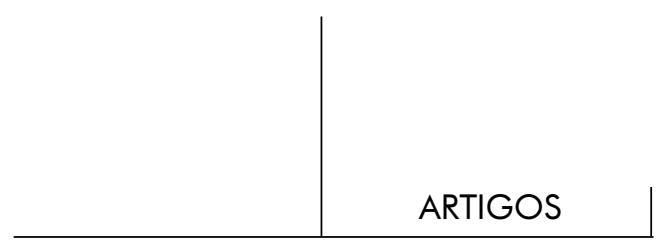

mo e refere-se à resiliência como um processo de reorganização psíquica frente ao mesmo (Anault, 2006; Bailly, 2006; Bertrand, 2006, Tychey \& Lighezzolo, 2006). O traumatismo não é constituído unicamente de um choque provocado por um agente externo, mas também pelas possibilidades de reação da pessoa frente ao mesmo (Hanus, 2006).

A resiliência implicaria, então, na capacidade do psiquismo para deter o traumático, originando novas condições psíquicas. $\mathrm{O}$ aparelho psíquico apresentaria um potencial criador de valor determinante para uma ação transformadora. Essa ação modificaria condições adversas e destinos pré-fixados (Zukerfeld, 2005). Por outro lado, a vulnerabilidade corresponderia a uma carência de recursos do eu, do qual fazem parte distorções cognitivas, dificuldade na elaboração de lutos e estilos de enfrentamento inadequados frente à situação traumática (Zukerfeld, 2005). Logo, não é possível quantificar a intensidade emocional de um evento traumático, pois esta depende da percepção intrapsíquica, que sofre influência dos aspectos mencionados. Assim, estudos qualitativos buscam compreender a diversidade das reações do indivíduo frente ao traumático, estabelecendo uma via de compreensão subjetiva da resiliência (Anault, 2006).

Outro ponto a destacar é o fato de a resiliência também ser investigada em situações de possível fragilidade psíquica que fazem parte do desenvolvimento humano típico, como a adolescência, a parentalidade e a velhice. Desse modo, sua investigação não estaria restrita a contextos graves e extremos como, por exemplo, violência e catástrofes (Bertrand, 2006). A psicanálise, de certo modo, instiga a pensar se este conceito está relacionado somente à resistência particular a traumas ou também às vicissitudes da existência (Chilland, 2006; Lemay, 2001).

Além disso, a psicanálise propõe-se a investigar a resiliência segundo duas vertentes teóricas distintas que, por sua vez, apresentam diferentes formas de análise desse conceito. A primeira tem por base a teoria das pulsões e a segunda a teoria das relações objetais, tendo como expoentes, entre outros autores, Bion (1991) e Winnicott (1956). Com base nos pressupostos dessas duas vertentes teóricas, serão descritas a seguir as possibilidades de investigação da resiliência.

Segundo a concepção da teoria das pulsões, a investigação da resiliência se dá por mecanismos de defesa utilizados no enfrentamento ao traumatismo (Anault, 2006; Bertrand, 2006). Essa abordagem tem por base tais mecanismos e analisa a maneira como estes são utilizados frente a uma situação traumática (Anault, 2006; Bertrand, 2006; Lemay, 2001; Tychey \& Lighezzolo, 2006). Os mecanismos de defesa são meios utilizados pelo ego para dominar, controlar e canalizar os perigos externos e internos. São estratégias inconscientes e involuntárias para regular a homeostase psíquica. As qualidades necessárias ao funcionamento resiliente pressupõem a flexibilidade dos modelos de ajustamento e de manejo de tais mecanismos, seguidas da criatividade nas formas de adaptação para vencer a adversidade (Anault, 2006). 
No processo resiliente prevalecem as defesas consideradas maduras e adaptativas, que contribuem para um bom estado de saúde mental. Entre estas, destacam-se a sublimação, o humor, a criatividade e o altruísmo, que servem de suporte às frustrações e auxiliam na gestão das pulsões agressivas presentes na situação traumática, condição necessária à manutenção do equilíbrio intrapsíquico (Anault, 2006; Tychey \& Lighezzolo, 2006). Já as defesas consideradas imaturas/primitivas, tais como a projeção, a passagem ao ato e o comportamento passivo-agressivo, contrapõem-se ao processo resiliente, por não possibilitarem ao ego a plasticidade e a flexibilidade necessárias para tal processo (Tychey \& Liguezzolo, 2006). Diante de tais questões, percebe-se que é possível falar em um funcionamento mais ou menos resiliente através dos mecanismos de defesa utilizados pelo ego. Estes se apresentam como estratégias adaptativas para suportar o trauma, bem como o sofrimento que o acompanha (Lemay, 2001; Tychey \& Liguezzolo, 2006).

Nessa perspectiva, a percepção do funcionamento mais ou menos resiliente do indivíduo se dá no trabalho psicoterápico, no qual se observa o seu efeito terapêutico em detrimento de circunstâncias desfavoráveis, mostrando-se como pulsão de vida e auxiliando o sujeito a progredir (Bailly, 2006). No trabalho psicoterápico o agente do trauma não poderá ser mudado, mas o impacto do choque poderá ser reelaborado (Hanus, 2006). De certo modo, o próprio processo terapêutico funcionaria como um fator promotor de resiliência.

Já de acordo com a segunda perspectiva, a teoria das relações objetais, a resiliência é construída nos primeiros anos de vida, em uma rede vincular caracterizada pela sustentação, apego e identificação, na intersubjetividade que se manifesta na relação com o outro (Zukerfeld, 2005). Dessa forma, a psicanálise propõe-se a investigar a resiliência a partir dos vínculos significativos desenvolvidos pelo indivíduo desde os primeiros anos de vida. Estes podem contribuir para a adaptação bem-sucedida frente ao traumatismo por serem importantes fontes de recursos intrapsíquicos (Bailly, 2006; Bertrand, 2006; Cyrulnik, 2004; Fonagy, 1999, 2000; Golse, 2006; Guedeney, 2006). Destacam-se, nessa perspectiva, especialmente as interações mãe-bebê.

A qualidade das interações precoces mãe-bebê possibilita a aquisição de recursos intrapsíquicos fundamentais, que colaboram para a promoção da resiliência no indivíduo. Tais recursos provêm de um processo intersubjetivo que visa proporcionar o desenvolvimento da segurança interna e da autonomia da criança (Cyrulnik, 2004; Golse, 2006; Guedeney, 2006). Para o entendimento de tal processo, serão apresentados alguns conceitos psicanalíticos que referem as interações precoces mãe-bebê como primordiais para a compreensão da construção da resiliência no indivíduo. Nesse sentido, destacam-se o conceito de rêverie (Bion, 1991), traumatismos hiperprecoces (Diatkine, 1994) e preocupação materna primária (Winnicott, 1956). 
O conceito de rêverie, proposto por Bion (1991), ilustra a importância do papel materno no processo intersubjetivo, que é essencial na construção do psiquismo do bebê. No início da vida extrauterina, a criança vivencia experiências somáticas e psíquicas, necessitando de um continente para projetá-las, de forma a transformá-las em pensamentos. A rêverie ocorre quando a mãe desempenha a função de continente para a criança, percebendo as experiências do filho e transformando-as em elementos pensáveis. A capacidade de rêverie se apresenta, ao mesmo tempo, como uma receptividade psíquica e uma capacidade de transformar em realidade as experiências da criança. Esta desempenha um papel fundamental no desenvolvimento psíquico da criança, preparando-a para dar conta de suas experiências com seu próprio aparelho psíquico (Houzel, 2005).

Com base no conceito descrito, e referindo-se também à teoria clássica do traumatismo, Diatkine (1994) descreveu os traumatismos hiperprecoces. A falha na interiorização da rêverie suficientemente boa (primeiro tempo da dinâmica do traumatismo) levaria o bebê a experimentar situações adversas posteriores (segundo tempo da dinâmica do traumatismo). Essa falha pode instalar a psicopatologia na criança, especialmente calcada nas experiências mais precoces da existência. Como exemplo, tem-se a depressão materna (Houzel, 2005). Uma mãe acometida de severa depressão no período pós-natal poderá ser capaz de proporcionar o cuidado físico necessário ao bebê. Porém, provavelmente será difícil e/ ou impossível que ela seja continente para as vivências instáveis do bebê, que são carregadas de fortes sensações, às vezes bastante desorganizadoras. A transformação dessas sensações do bebê, pela mãe, em algo que tenha sentido para ele e que, por conseguinte, o auxilie na assimilação psíquica de tais sensações, fica comprometida, diante do prejuízo da capacidade de rêverie da mãe (Houzel, 2005). A partir desse comprometimento da mãe, o bebê pode vivenciar traumatismos hiperprecoces.

Por fim, o terceiro conceito fala da capacidade de empatia da mãe para identificar as necessidades da criança. De fato, os cuidados maternos, importante aspecto da relação materno-filial, pressupõem a identificação da mãe com o bebê. A elaboração dessa capacidade empática para com o bebê, que ocorre gradativamente ao longo da gravidez, faz com que a mãe evolua para um estado específico denominado preocupação materna primária (Winnicott, 1956). Esse estado permite a ela atingir progressivamente um grau de sensibilidade aumentada ao longo das semanas anteriores ao parto. Desse modo, a mãe direciona toda a sua atenção para o bebê, renunciando a alguns interesses pessoais nesse momento. Essa renúncia materna em prol do provimento das necessidades do bebê busca dar suporte inicial à estruturação do ego da criança, propiciando a ela o sentimento de existir enquanto ser. Deve-se destacar, no entanto, que nem todas as mães 
conseguem desenvolver tal preocupação, o que poderá acarretar em prejuízos para o desenvolvimento infantil.

A partir dos conceitos referidos, é possível analisar a resiliência como uma construção psíquica, com início no contexto da relação pais-bebê (Golse, 2006). Tais relações precoces estabelecem estilos relacionais mais ou menos resilientes para a criança. Destaca-se que uma mãe afetivamente suprida e com suporte conjugal, familiar e social tem mais condições de compreender e atender as necessidades de seu bebê (Cyrulnik, 2004). Isso possibilitará uma sustentação inicial à criança no enfrentamento do contexto social, no qual estão presentes, por vezes, diversas outras adversidades (Cyrulnik, 2004).

\section{Considerações finais}

O presente trabalho teceu algumas considerações sobre o conceito resiliência, destacando o seu entendimento na perspectiva psicanalítica, bem como possibilidades de investigação nessa teoria. Ressalta-se que a psicanálise analisa esse conceito de uma forma particular, a partir dos aspectos intrapsíquicos do indivíduo, propondo com isso uma nova interpretação da resiliência. Dessa forma, busca ampliar o conhecimento e as formas de investigação sobre esse conceito ainda novo no campo da saúde.

Independentemente das diferentes formas de análise da resiliência propostas pela psicanálise, percebe-se que essa perspectiva teórica e clínica analisa esse conceito como um processo, e não como uma característica mais estável do indivíduo. De outra forma, não é possível prever quem será ou não resiliente, mas, sem dúvida, é possível ter indicativos, a partir da análise dos aspectos intrapsíquicos do indivíduo frente a situações adversas, da sua capacidade de resiliência. Pensa-se que o foco individual e subjetivo da resiliência não descarta a importância da análise do meio familiar e social no seu desenvolvimento, uma vez que, como visto, também constituem aspectos importantes nessa construção individual. De fato, pensar a resiliência como uma construção psíquica com início na relação pais-bebê demonstra a importância das relações precoces no seu estabelecimento, colocando mãe/pai como os primeiros tutores do desenvolvimento da resiliência na criança (Cyrulnik, 2004; Golse, 2006). Entretanto, quando essas primeiras relações não se organizam de uma forma satisfatória, o trabalho terapêutico, que pode ser realizado com adultos, adolescentes e crianças, contribui para a promoção da resiliência. Esse trabalho, a partir da identificação da influência dos aspectos intrapsíquicos na promoção da resiliência, propicia uma nova condição psíquica ao indivíduo no enfrentamento de situações adversas. 


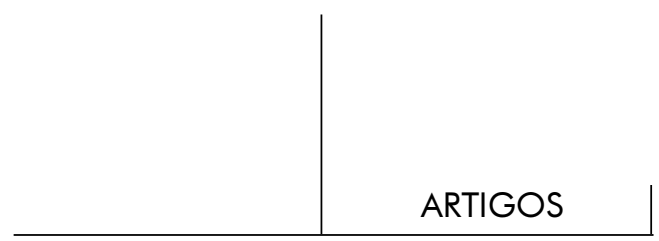

Agradecimentos: Agradecemos a Bernard Golse (Université René Decartes, Paris V; Hôpital Necker Enfants Malade, Paris), que gentilmente enviou e sugeriu alguns textos para este trabalho.

\section{Referências}

Anault, M. (2006). La résilience au risque de la psychanalyse ou la psychanalyse au risque de la résilience. In B. Cyrulnik \& P. Duval (Orgs.), Psychanalyse et Résilience (pp. 77-104). Paris: Odile Jacob.

Bailly, L. (2006). Résilience et psychanalyse. In B. Cyrulnik \& P. Duval (Orgs.), Psychanalyse et Résilience (pp. 223-234). Paris: Odile Jacob.

Bertrand, M. (2006). Résilience et traumatismes: un point de vue psychanalytique. In B. Cyrulnik \& P. Duval (Orgs.), Psychanalyse et Résilience (pp. 205-222). Paris: Odile Jacob.

Bion, W. R. (1991). O aprender com a experiência. Rio de Janeiro: Imago.

Bourguignon, O. (2006). Perspectives théoriques et réalites humaines. In B. Cyrulnik \& P. Duval (Orgs.), Psychanalyse et Résilience (pp. 105-126). Paris: Odile Jacob.

Bronfenbrenner, U. \& Morris, P. (1998). The ecology of developmental processes. In W. Damon \& R. M. Lerner (Orgs.), Handbook of child psychology: Theoretical models of human development (pp. 933-1028). New York: John Wiley.

Chillan, C. (2006). Réticence à propôs de la résilience. In B. Cyrulnik \& P. Duval (Orgs.), Psychanalyse et Résilience (pp. 248-263). Paris: Odile Jacob.

Cyrulnik, B. (2004). Os patinhos feios. São Paulo: Martins Fontes.

Delage, M. (2002). Aide à la résilience familiale dans les situations traumatiques. Thérapie familiale, Genéve, 23(3), 269-287.

Diatkine, R. (1994). A criança no adulto ou a eterna capacidade de sonhar. Lisboa: Instituto Piaget.

Fonagy, P. (1999). Persistências transgeneracionales del apego: una nueva teoria. Aperturas Psicoanalíticas - Revista Internacional de Psicoanálisis, Madri, 3. Recuperado de <http://www.aperturas.org/articulos.php?id=0000086\&a= Persistencias-transgeneracionales-del-apego-una-nueva-teoria>

Fonagy, P. (2000). Apegos patológicos y acción terapêutica. Aperturas Psicoanalíticas - Revista Internacional de Psicoanálisis, Madri, 4. Recuperado de <http:// www.aperturas.org/articulos.php?id=0000104\&a=Apegos-patologicos- $y$-accionterapeutica>.

Freud, S. (1996). Projeto para uma psicologia científica. In Edição Standard Brasileira das Obras Psicológicas Completas de Sigmund Freud (pp. 387-529). Rio de Janeiro: Imago. (Trabalho original publicado em 1895).

Freud, S. (1996). Além do princípio de prazer. In Edição Standard Brasileira das Obras Psicológicas Completas de Sigmund Freud (pp. 105-154). Rio de Janeiro: Imago. (Trabalho original publicado em 1920). 
Golse, B. (2003). Sobre a psicoterapia pais-bebê: narratividade, filiação e transmissão. São Paulo: Casa do Psicólogo.

Golse, B. (2006). Le pédopsychiatre - psychanalyste face au concept de résilience. La résilience avant l'après-coup. In B. Cyrulnik \& P. Duval (Orgs.), Psychanalyse et Résilience (pp. 61-76). Paris: Odile Jacob.

Guedeney, A. (2006). L'attachement el la résilience: théorie, clinique et politique sociale. In B. Cyrulnik \& P. Duval (Orgs.), Psychanalyse et Résilience (pp. 155-156). Paris: Odile Jacob.

Hanus, M. (2006). Freud et Prométhée, un abord psychanalytique de la resilience. In B. Cyrulnik \& P. Duval (Orgs.), Psychanalyse et Résilience (pp. 168-178). Paris: Odile Jacob.

Houzel, D. (2005, set.). Influência de fatores familiares sobre a saúde mental de crianças e de adolescentes. Revista Latinoamericana de Psicopatologia Fundamental, São Paulo, VII(3), 443-479.

Laranjeira, C. A. S. de J. (2007, jul.set.). Do invulnerável ser ao resiliente envelhecer: revisão de literatura. Psicologia: teoria e pesquisa, Brasília, 23(3), 327-332.

Lemay, M. (2001). La résilience avant la violence. Revue Québécoise de Psychologie, 22(1), 135-148.

Lindström, B. (2001, abr.). O significado de resiliência. Adolescência Latinoamericana, Porto Alegre, 2(3), 133-137.

Paludo, S. dos S. \& Koller, S. H. (2007, jan.abr.). Psicologia positiva: uma nova abordagem para antigas questões. Paidéia, Ribeirão Preto, 17(36), 9-20.

Sapienza, G. \& Pedremônico, M. R. M. (2005, maio.ago). Risco, proteção e resiliência no desenvolvimento da criança e do adolescente. Psicologia em Estudo, Maringá, 10(2), 209-216.

Seligman, M. \& Csikszentmihalyi, M. (2000, jan.), Positive Psychology: an introduction. American Psychologist, 55(1), 5-14.

Souza, M. T. S. de \& Cerveny, C. M. de O. (2006). Resiliência psicológica: revisão da literatura e análise da produção científica. Revista Interamericana de Psicologia, 40(1), 119-126.

Tychey, C. de \& Lighezzolo, J. (2006). La résilience au regard de la psychologie clinique psychanalytique. In B. Cyrulnik \& P. Duval (Orgs.), Psychanalyse et Résilience (pp. 127-154). Paris: Odile Jacob.

Zukerfeld, R. (2005, jun). Psicanálise, psicossomática e medicina: sobre a vulnerabilidade e a resiliência. Revista da Sociedade de Psicologia do Rio Grande do Sul, Porto Alegre, 4(1), 7-17.

Yunes, M. Â. M. (2003). Psicologia positiva e resiliência: o foco no indivíduo e na família. Psicologia em Estudo, Maringá, 8 (n. especial), 75-84.

Yunes, M. Â. M. e Szymanski, H. (2006). O estudo de uma família "que supera as adversidades da pobreza": um caso de resiliência em família? Psicodebate: Psicologia, Cultura y Sociedad, Palermo, 7, 119-139. 


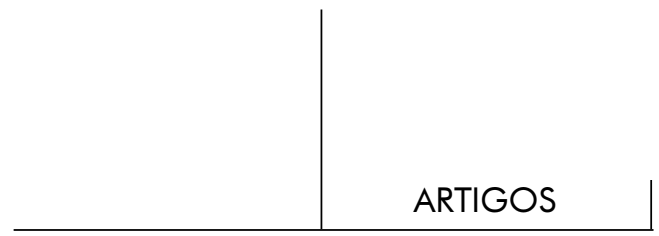

Walsh, F. (1996, sept.). The concept of family resilience: crisis and challenge. Family Process, 35(3), 61-181.

Winnicott, D. W. (2000). Da pediatria à psicanálise - Obras Escolhidas. Rio de Janeiro: Imago. (Trabalho original publicado em 1956).

\section{Resumos}

(Resilience and psychoanalysis: theoretical aspects and possibilities for research)

Resilience is a concept in the field of health that refers to people's resources in the face of adversity. It is an important and useful concept that deserves debate in different theoretical and clinical contexts. This study presents the theoretical bases of this concept and discusses the possibilities of analyzing resilience from a psychoanalytic perspective. Resilience here is approached by analyzing the intrapsychic aspects of subjects, enabling new interpretations of the concept.

Key words: Resilience, psychoanalysis, psychic aspects, mental health

(Résilience et psychanalyse: aspects théoriques et possibilités d'investigation)

La résilience est un concept qui relève du domaine de la santé et qui analyse les ressources saines de l'individu que celui-ci utilise dans des situations adverses. Discuter ses possibilités dans de différents contextes théoriques et cliniques représente un débat constructif et nécessaire. Notre étude présente les bases de ce concept et discute les possibilités d'analyse de la résilience du point-de-vue de la psychanalyse. Cette perspective examine la résilience en analysant les aspects intrapsychiques de l'individu, fournissant ainsi une nouvelle interprétation du concept.

Mots clés: Résilience, psychanalyse, aspects intrapsychiques, santé mentale

(Resiliencia y Psicoanálisis: Aspectos Teóricos y Posibilidades de Investigación)

La resiliencia es un concepto del campo de la salud que analiza los recursos de salud del individuo frente a situaciones adversas. Discutir sus posibilidades en los diferentes contextos teóricos y clínicos es un debate necesario y constructivo. Este estudio presenta los supuestos de este concepto y discute las posibilidades de análisis de la resiliencia desde una perspectiva psicoanalítica. Esta perspectiva investiga la resiliencia por medio del análisis de los aspectos intrapsíquicos de la persona, proporcionando una nueva interpretación de este concepto.

Palabras clave: Capacidad de recuperación, psicoanálisis, aspectos intrapsíquicos, salud mental 
(Resilienz und Psychoanalyse: theoretische Aspekte und Untersuchungsmö glichk

en)

Resilienz ist ein Begriff aus dem Gesundheitswesen, der die gesunden Fähigkeiten des Individuums gegenüber widriger Situationen beschreibt. Es ist eine konstruktive und notwendige Auseinandersetzung, ihre Fähigkeiten in verschiedenen theoretischen und klinischen Kontexten zu besprechen. Dieser Beitrag stellt die Voraussetzungen dieses Begriffs vor und diskutiert die Analysemöglichkeiten von Resilienz im Rahmen der psychoanalytischen Perspektive. Diese Perspektive untersucht die Resilienz durch die Analyse der intra-psychischen Aspekte des Individuums und ermöglicht dadurch eine neue Begriffsauffassung.

Schlüsselwörter: Resilienz, Psychoanalyse, intrapsychische Aspekte, geistige Gesundheit

Citação/Citation: Cabral, S. A. \& Levandowski, D. C. (2013, março). Resiliência e psicanálise: aspectos teóricos e possibilidades de investigação. Revista Latinoamericana de Psicopatologia Fundamental, 16(1), 42-55.

Editor do artigo/Editor: Prof. Dr. Manoel Tosta Berlinck

Recebido/Received: 3.4.2012/ 4.3.2012 Aceito/Accepted: 19.7.2012/7.19.2012

Copyright: (C) 2009 Associação Universitária de Pesquisa em Psicopatologia Fundamental/ University Association for Research in Fundamental Psychopathology. Este é um artigo de livre acesso, que permite uso irrestrito, distribuição e reprodução em qualquer meio, desde que o autor e a fonte sejam citados / This is an open-access article, which permits unrestricted use, distribution, and reproduction in any medium, provided the original author and source are credited.

Financiamento/Funding: As autoras declaram não ter sido financiadas ou apoiadas / The authors have no support or funding to report.

Conflito de interesses/Conflict of interest: As autoras declaram que não há conflito de interesses / The authors declare that has noconftict fofinterest. 


\section{Stela Araújo Cabral}

Psicóloga Clínica; Mestre em Psicologia Clínica pela Universidade do Vale do Rio dos Sinos - Unisinos (São Leopoldo, RS).

Rua José Bonifácio, 556/301

93010-180 São Leopoldo, RS, Br

e-mail: stela.c@hotmail.com

\section{Daniela Centenaro Levandowski}

Psicóloga, Mestre em Psicologia do Desenvolvimento e Doutora em Psicologia pela Universidade Federal do Rio Grande do Sul - UFRGS (Porto Alegre, RS, Br), com Pós-Doutorado em Psicologia (PUC-RS). Docente e Pesquisadora do Departamento de Psicologia e do Programa de Pós-Graduação em Ciências da Saúde da Universidade Federal de Ciências da Saúde de Porto Alegre (UFCSPA). Pesquisadora do CNPq e da FAPERGS. UFCSPA

Rua Sarmento Leite, 245 Sala 207 90050-170 Porto Alegre, RS, Br

e-mail: danielal@ufcspa.edu.br 